\title{
An optimized scalable multi-ant colony system for multi-depot vehicle routing problems using a reactive multi-agent system
}

\author{
GUIA SANA SAHAR ${ }^{1,2}$, KAZAR OKBA $^{2}$, LAOUID ABDELKADER ${ }^{1,3}$, \\ Yagoub Mohammed Amine ${ }^{1}$, Reinhardt EuleR ${ }^{4,5}$, AHCÈne BounCEuR ${ }^{4,5}$, \\ MOHAMMAD HAMMOUDEH ${ }^{4,5}$ \\ El Oued University, ALGERIA ${ }^{1}$ \\ Informatique Intelligent Laboratory, Biskra University, ALGERIA ${ }^{2}$ \\ LIMED Laboratory, University of Bejaia, 06000, Bejaia, ALGERIA ${ }^{3}$ \\ Department of Computing and Mathematics, Manchester Metropolitan University, UNIITED KINGDOM ${ }^{4}$ \\ Lab-STICC UMR CNRS 6285, Université de Bretagne Occidentale, \\ 20 Avenue Victor Le Gorgeu, 29238 Brest, FRANCE 5
}

\begin{abstract}
The multi-depot vehicle routing problem is a variant of the vehicle routing problem that tries to minimize the total cost of providing the service from several depots to satisfy several client demands. This paper presents a multi-ant colony system to solve the multi-depot vehicle routing problem using a reactive agent-based approach. This approach is designed to effectively solve the problem, in which each reactive agent is inspired by modeling the behavior of the ant. We define two types of reactive agents whose behavior differs in the use of two kinds of pheromone trail. In order to refer to the two phases of the execution process, i.e., the assignment phase and the routing phase, every reactive agent cooperates with others to provide a scalable solution for the overall problem. The solution of the multi-depot vehicle routing problem is beneficial and helpful for many real applications. The performance evaluation of the proposed approach is done using instances from the literature, and the results obtained demonstrate good performance when compared with other approaches.
\end{abstract}

Key-Words: - Multi-Depot Vehicle Routing Problem, Ant Colony, Reactive Multi-Agent System.

Received: March 30, 2021. Revised: August 1, 2021. Accepted: August 7, 2021. Published: August 17, 2021.

\section{Introduction}

The Multi-Depot Vehicle Routing Problem (MDVRP) is an extension of the Vehicle Routing Problem (VRP) where vehicles start from one of multiple depots. Each depot contains a specific fleet of vehicles, and each vehicle must end at its start depot [1] to serve a group of dispersed clients.

Similar to supply chain networks, the multi-depot vehicle routing problem MDVRP consists of several depots and multiple delivery points, that allocates vehicles to the desired destinations in an optimal way, a problem which requires robust approaches to find best solutions.

In the early 1990s, Marco Dorigo and colleagues [2] developed an Ant colony optimization metaheuristic . Ant Colony Optimization (ACO) solutions are competitive with existing metaheuristic approaches by providing significant quality solutions to hard Combinatorial Optimization (CO) problems such as the vehicle routing problem with respect to computation times.

During the execution of an ACO algorithm, a colony of artificial ants (simple agents), use distributed numerical information called pheromone trails to probabilistically construct solutions. Therefore, the ants communicate indirectly through pheromone trails and adapt to reflect their search experience of the problem being solved [3].

Using a multi-agent system in the implementation of meta-heuristic optimization frameworks facilitates the hybridization of metaheuristics, flexibilizes the development of hybrid meta-heuristics and allows simultaneous exploration of different regions of the search space [4]

Multi-agent systems were used as a new approach for the design of ACO meta-heuristics solving the MDVRP.

Exploiting Reactive Multi-Agent Systems (RMAS), the behavior of ant colonies can be simulated when they are looking for food to find good paths through graphs.

A Reactive Multi-Agent System (RMAS) is designed for solving problems or modelling the behavior of complex systems. An RMAS provides a good supervision system by exchanging information more efficiently and offering faster response in 
terms of time [5]. The summary of the paper is as follows: Section 2 represents previous work on the problem under study. Section 3 describes the reactive agent-based approach modelling the multiant colony system for solving the multi-depot vehicle routing problem MDVRP. Experiments are provided in Section 4. Finally, Section 5 ends the paper with conclusion and futur work.

\section{Literature Review}

Among the wide spectrum of transportation research, the VRP and its various models have attracted considerable attention. This is principally the result of the supplemental constraints caused by real-world problems, the possibility of cost savings and the opportunity of service improvement for organizations involved in distribution networks.

The multi-depot vehicle routing problem MDVRP is one of the common extensions of the vehicle routing problem VRP, that consists in serving clients from several depots using a fleet of a vehicle for each depot. In this context, many metaheuristic approaches have been proposed, such as genetic algorithms GA, tabu search TS, ant colony optimization ACO. Meta-heuristic algorithms are able to generate a close to the best solution within appropriate time. In recent years, there has been an emergence of hybridization techniques which combine meta-heuristic methods with other optimization algorithms [6 - 9].

In [10], two methodologies are proposed solving the MDVRP with a Fixed vehicle Distribution (MDVRPFD): the two-stage approach, in which assignment and routing subproblems are solved separately, and the single-stage approach that combines the assignment and the routing to solve them simultaneously. Then a specific segmentation algorithm is applied to determine the lowest possible cost cycles [11]. In [12], the geometrybased genetic clustering algorithm is proposed for assigning clients to the depots in the MDVRP. Based on the well-known tabu search TS, Escobar et al. [13] described a hybrid tabu search algorithm for the MDVRP. Subsequently, Li et al. [14] proposed an adaptive local search algorithm based on a hybrid genetic solution to solve the MDVRP with timewindows within shared depot resources. More recently, Bezerra et al. [15] propose a solution for the MDVRP through variants of the Variable Neighbourhood Search algorithm (VNS) with Adaptive Local Search (ALS).

The Ant Colony System (ACS) is one of the most studied meta-heuristic algorithms for solving
VRP [16], as it has been proven to be very effective in solving different types of VRP. Tan et al. proposed in [17] an ant colony system to improve the VRP solution with time windows. In [18], a Multi-Ant Colony System (MACS) is used to solve the VRP with Backhaul (VRPB), in which two sorts of ants are used to build the solution. A metaheuristic based on an ant colony system is proposed in [19] to resolve the Multi-Depot Green Vehicle Routing Problem (MDGVRP). The proposed distribution network uses Alternative Fuel- Vehicles (AFVs) to treat the obstacles caused by the restricted number of the Alternative Fuel Stations. Li et al. in their work [20] solve the Multi-Depot Green VRP (MDGVRP) using a new technique for updating the development of pheromone in the ordinary ACO algorithm, called (IACO) for Improved ACO, that aims to efficiently increase revenue and reduce each of routing cost, time and emission. Stodola [7] combines the ACO algorithm introduced in [21] with the principles of simulated annealing and a deterministic topical optimization process. In [22], an ant colony system with twopheromone trail solutions is proposed for the Heterogeneous Vehicle Routing Problem with Time Windows and Multiple types of Products containing Incompatibility Constraints (HVRPTWMPIC). The proposed model in this research aims at minimizing cost and the number of vehicles per depot.

As shown in Table 1, many ACO meta-heuristic approaches for the multi-depot vehicle routing problem MDVRP have been proposed. These methods aim to minimize the total cost of providing service, to maximize revenue and especially to minimize cost, time and distance. The proposed approach in this research aims to reduce the total distance travelled from depots to serve clients, using a minimum size vehicle fleet.

Many proposals have been implemented to solve different variations of the vehicle routing problem VRP using multi-agent systems [5]. In [23], the authors present an interactive distributed approach that proceeds by interaction between agents and which is focused on a particular multi-agent model based on a coalition formation called Coal-VRP (Dynamic generation of Coalition for solving a VRP) to solve a Vehicle Routing Problem with Time Windows (VRPTW). In [24], the authors proposed a multi-agent enhanced model to solve a vehicle routing problem with time windows VRPTW based on the internal behavior of agents and the coordination among the agents using an 


\begin{tabular}{|c|c|c|c|c|c|c|c|c|}
\hline \multirow[t]{2}{*}{ Year } & \multirow[t]{2}{*}{ Authors } & \multicolumn{6}{|c|}{ Objective type } & \multirow[t]{2}{*}{ Solution method } \\
\hline & & Cost & Time & Income & emission & $\begin{array}{c}\text { Number of } \\
\text { vehicles }\end{array}$ & Distance & \\
\hline 2011 & Yu et al [27] & * & & & & & & Parallel Improved ACO \\
\hline 2016 & Wang et al [9] & * & & & & & & Hybrid ACO \\
\hline 2016 & Gao et al [28] & * & & & & & * & Improved ACO \\
\hline 2017 & Jabir et al [8] & * & & & * & & & Hybrid ACO \\
\hline 2017 & Ma et al [29] & * & & & & & & Improved ACO \\
\hline 2018 & Zhang et al [19] & & & & * & & * & Hybrid ACO \\
\hline 2019 & Yongbo et al [20] & * & * & * & * & & & Improved ACO \\
\hline \multirow[t]{2}{*}{2020} & Stodola [7] & & * & & & & * & Hybrid ACO \\
\hline & This study & * & * & & & * & * & Multi ACS \\
\hline
\end{tabular}

Table 1: Summary of relevant MDVRP research using ACO meta-heuristic

improved Contract-Net Protocol (CNP). An agent-oriented self-organization model to solve a dynamic version of VRPTW has been proposed in [25] focusing on a space-time representation of agents' action zones. Hassen et al. [26] proposed a dynamic route plan approach based on a multi-agent system for the VRPTW related to the home health care routing and scheduling problem by adopting the Artificial Immune Algorithm (AIS) to generate the optimal route. In [22], an approach based on multi-agent technologies dedicated to the MDVRP with Time Windows (MDVRPTW) has been proposed. To accelerate the interaction between agents and to limit the intensity of negotiations, a structure involving the Delaunay triangulation during scene analysis is suggested.

Focusing solely on meta-heuristics is relatively restricted when dealing with hard combinatorial optimization problems. Concept combinations from different approaches may provide efficient results with more flexibility, such as techniques for optimization that combine artificial intelligence with operational research into the solution process. To this end, this paper is a continuity of our work published in [30], but here we propose a reactive multi-agent system to model a multi-ant colony system for solving the MDVRP. Therefore, it should be more intelligent to look for solving the multidepot vehicle routing problem MDVRP and the scalability is required to benefit from the autonomy characters and the auto-adaptability of agents. Furthermore, our paper includes a multi-ant colony system into the multi-depot vehicle routing problem MDVRP domain by focusing on a new way to update pheromone that produces quietly better solutions. According to Table 1, most of the ACO meta-heuristics that are proposed for the multi-depot vehicle routing problem MDVRP focus on the objective of reducing travel time, transport cost and travel distance without including the objective of minimizing the number of vehicles. Therefore, the approach proposed in this study aims to satisfy this latter objective together with the objectives of minimizing time, cost and distance.

\section{Problem Formulation}

Dantzig and Ramser introduced the vehicle routing problem VRP as a generalization of the Travelling Salesman Problem (TSP) introduced by Flood [31]. In the domain of combinatorial optimization, the vehicle routing problem VRP is one of the most challenging research problems. The problem was defined more than 40 years ago, and it involves designing an optimal set of routes for a fleet of vehicles to serve a specific set of clients. 
The fleet of vehicles is situated at a central depot, every vehicle has a certain capacity and each client has a given demand. The aim is to minimize the total distance travelled to serve a geographically dispersed group of clients [32]. In the literature, several algorithms are available for the vehicle routing problem VRP. Due to its NP-hardness, exact methods trend to poor performance on large instances, which explains why so many heuristics have been developed [33].

These enclose construction and improvement procedures or two-phase approaches for classical heuristics, and meta-heuristics such as simulated annealing, tabu search, variable neighbourhood search or evolutionary algorithms [33].

MDVRP is a well-known generalization of the vehicle routing problem VRP. In this variant, each client is visited by a vehicle operated from one of several depots. In the standard multi-depot vehicle routing problem MDVRP, each vehicle route must end at its start depot.

This section deals with the mathematical formulation of the problem. First, we have the following data:

- a set of depots,

- a set of commands,

- a fleet of vehicles,

with constraints given by:

- the vehicle capacity,

- the distance between clients and depots.

The objective is to reduce the total distance travelled from depots to serve clients, using a fleet of vehicles for every depot.

The issue of solving the MDVRP faces several design challenges which can be resumed as follows:

- Assignment of clients to depots: this includes clustering a group of clients to a specific depot through which services are provided to clients.

- Routing and sequencing of service: this involves determining several routes for each depot, by assigning each client to a unique route without exceeding the capacity of the vehicle, and finally, determining a series of clients on every vehicle route.

The multi-depot vehicle routing problem can have the following description [1]:

- $\mathrm{D}, \mathrm{R}, \mathrm{V}$ denote the sets of depots, of clients and of vehicles, respectively,

- $\mathrm{S}$ is the number of all depots,

- $\mathrm{N}$ is the number of all clients,

- $\mathrm{M}$ is the number of vehicles for each depot,

- $\mathrm{Q}(\mathrm{k})$ is the capacity of vehicle $\mathrm{k}$,
- $\quad \mathrm{E}$ is the edge set between clients and depots, $e_{i j} \in E, i, j \in D \cup R$,

- bi is the demand of client $i$,

- $c_{i j}$ is the cost of transporting one unit from depot $i$ or client $i$ to client $j$, or from client $\mathrm{i}$ to depot $\mathrm{j}$ or client $\mathrm{j}, i, j \in D \cup R$,

- $x_{i j}^{k}$ is 1 , if vehicle $\mathrm{k}$ travels from $\mathrm{i}$ to $\mathrm{j}$, $i, j \in D \cup R ; 0$, otherwise.

- $y_{i k}$ is 1 , if vehicle $\mathrm{k}$ offers service for $\mathrm{i} ; 0$, otherwise.

Using these definitions, the problem under study can be formulated as follows:

$$
\min \sum_{i \in D \cup R} \sum_{j \in D \cup R} c_{i, j} \sum_{k \in V} x_{i, j}^{k}
$$

subject to:

$$
\begin{gathered}
\sum_{j \in D \cup R j \neq i} \sum_{k \in V} x_{i, j}^{k}=1, \quad i \in R \\
\sum_{i \in D} b_{i} y_{i k} \leq Q(k), k \in V \\
\sum_{i \in D} \sum_{j \in R} x_{i, j}^{k} \leq 1, k \in V \\
\sum_{i \in D \cup R} x_{i, j}^{k}=\sum_{j \in D \cup R} x_{j, i}^{k} \leq 1, k \in V
\end{gathered}
$$

Equation (1) represents the objective function whose goal is to minimize the total travel cost. Equation (2) makes sure that every client is allocated to a single route. Equation (3) is the capacity constraint set for vehicles. Equation (4) assures that each client can be served only once. Equation (5) ensures that all delivery vehicles must return to the original depot after finishing their task.

\section{Proposed solution}

The vehicle routing problem VRP with its various variants remains at the heart of many service operations and organizations involved in the scheduling of client service, and the routing of service vehicles has to face a lot of challenges. A lots of researchers aim to improve the solution quality by using an ACO algorithm, which probabilistically solves computational problems.

We use a Reactive Multi-Agent System RMAS for modelling the Multi-Ant Colony System (MACS). The multi-ant approach is based on a "cluster first and route second" procedure of the multi-depot vehicle routing problem MDVRP. More precisely, two types of ants are used to construct the solution, where each ant is modelled by a reactive agent. Ants of the first type are used to allocate clients to depots and ants of the second type are 
used to construct the route for a specific depot that is assigned to a set of clients, namely, to solve the underlying vehicle routing problem. The execution process phases are: the setting phase, the assignment phase and the routing phase (see Figure 1).

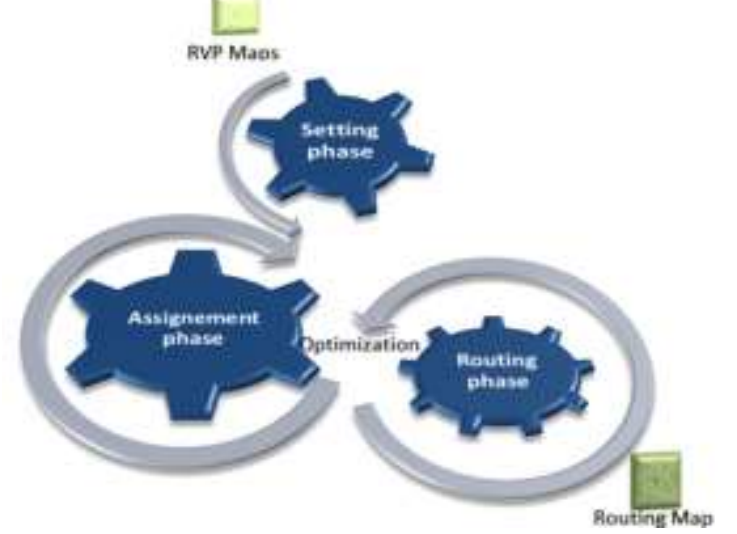

Fig.1 Architecture of the proposed approach.

As there is more than one depot in the multidepot vehicle routing problem MDVRP, the algorithm uses several ant colonies at the same time. Every depot creates a colony from which ants start visiting clients. This approach introduces two types of ants modelled by the reactive agent: the assignment_ant_agent and the route_ant_agent referring to the assignment phase, and the routing phase, respectively. Therefore, we define two pheromone trail intensities: $\tau 1$ as the pheromone trail of the assignment_ant_agent, and $\tau 2$ as the pheromone trail of the route_ant_agent.

An overview of the proposed algorithm is shown in Figure 2 and a more detailed description of the algorithm is provided in the next subsection.

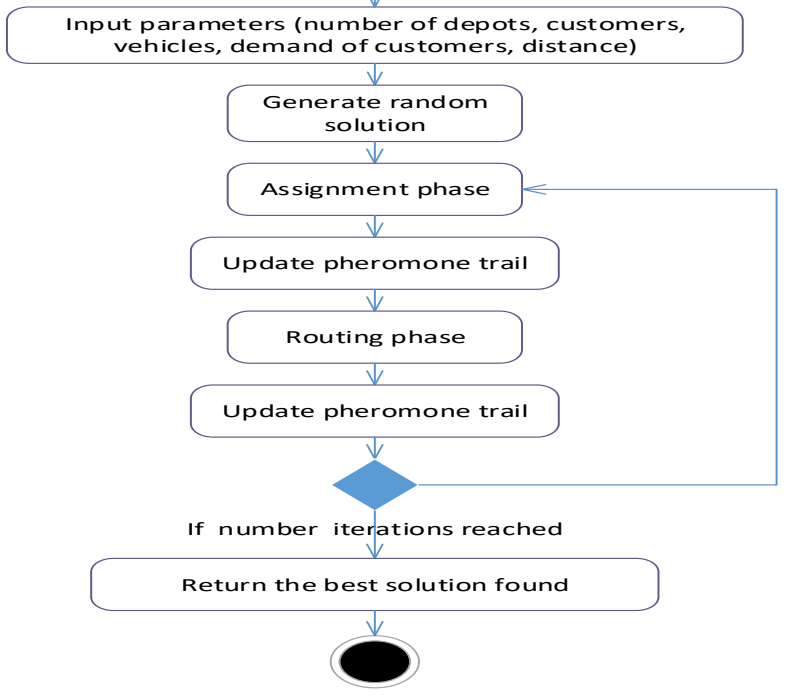

Fig.2 Activity diagram of the multi-ant colony system algorithm.
This algorithm is a multi-agent system which has been inspired by the behavior of ants exploring their environment to find food. Thus, each reactive agent is an artificial ant, and a large number of reactive agents cooperate to build solutions via low-level based communication and collaborative behavior. According to Figure 2, reactive agents randomly explore the environment and generate a random solution. In the next phase, clients are assigned to depots after creating a colony of assignment_ant_agents to this end. Hereafter, a colony of route ant agents is created to construct several routes for each depot, and to determine a sequence of clients on each vehicle route. The assignment and routing phases are repeated until a fixed number of iterations is reached. Finally, the best solution found is returned.

\subsection{Setting phase}

According to Figure 2, after entering the model parameters, i.e., client's map and commands, depots map, vehicle capacities and distance setting, we generate an initial solution by assigning clients to their nearest depot, and we apply a single iteration of the routing phase algorithm for the VRP of each depot.

The pheromone trail intensities are initialized as follows:

- The $\tau 1$ pheromone trail of the assignment_ant_agent is initialized after generating the first solution by $\tau 1_{i j}=$ $c_{i j} /$ best_local_cost $_{d_{i}}$ in which $\tau 1_{i j}$ is the pheromone trail between depot $\mathrm{i}$ and client $\mathrm{j}, c_{i j}$ is the cost of transportation between depot $\mathrm{i}$ and client $\mathrm{j}$, and best_local_cost $d_{i}$ is the best cost of transportation of the route of the depot $i$ which was found in this generation.

- The $\tau 2$ pheromone trail of the route_ant_agent is initialized first before generating the initial solution by a random value. After generating the first solution $\tau 2$ which is reinitialized by $\tau 2=$ best_local_cost $_{d_{i}} /$ best_global_cost in which best_global_cost is the best solution found in the current generation.

\subsection{Assignment phase}

The first phase after the generation of the first solution and the initialization of the pheromone trail is the assignment of clients to depots which is realised by assignment_ant_agents. The algorithm uses several ant colonies at the same time. A colony of assignment_ant_agents is created for each depot 

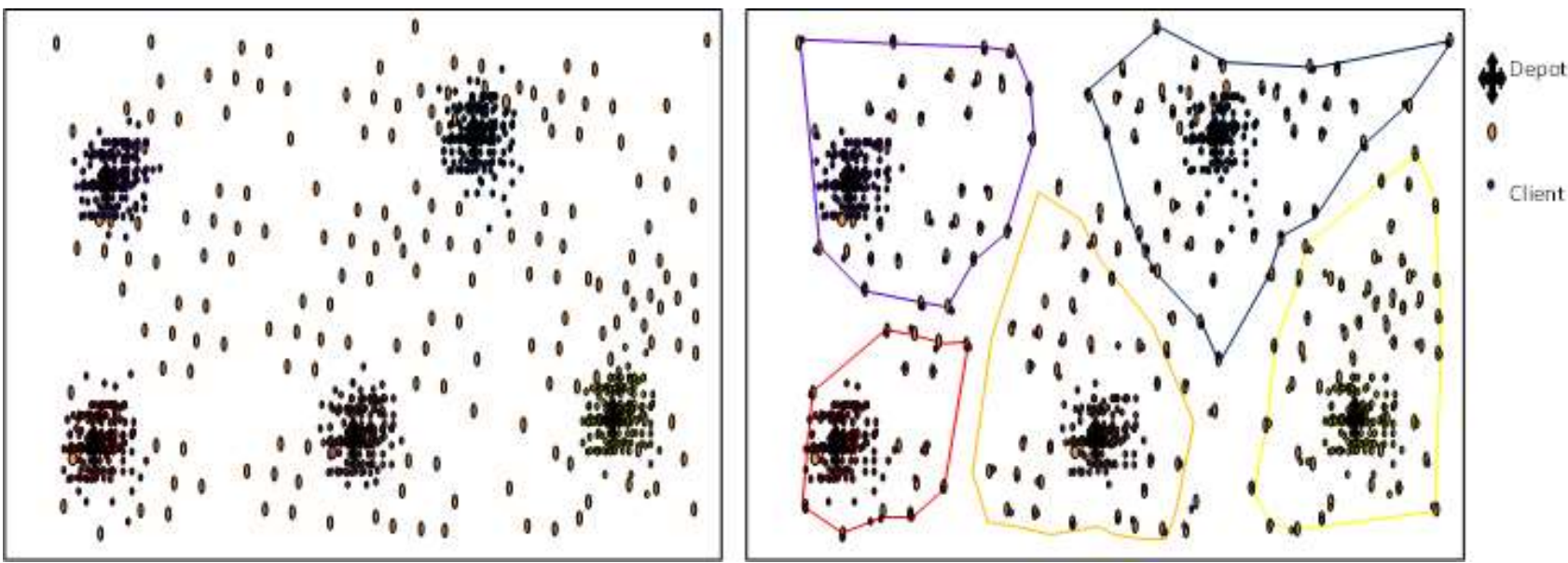

Fig. 3 An overview on the result of the assignment phase.

and it runs in a parallel and concurrent manner to choose the appropriate clients. In each iteration of the algorithm, we generate a new solution depending on the behavior of the assignment_ant_agents. At the end, we will keep the solution that minimizes the total distance travelled.

The result of the assignment phase is shown in Figure 3.

\section{Algorithm 1. Assignment}

Input Map clients and setting ( $\mathrm{x}$ and $\mathrm{y}$ coordinate of each client )

Output Assigned Map clients

\section{In parallel}

2

3

4

5

6

7

8

9

10

11

12

13

14

15

16

17

18
For each depot

Create a colony of assignment_ant_agents

Repeat

Create ants

For each ant

Affect the capacity of vehicles

Begin from the depot

Repeat

Calculate ant's probabilities to visit not affected clients

if $(\mathrm{C}=$ select a client determined by the probability and demand of client) then

Move to the client

Decrease the demand of client from the capacity of the ant else

ant will die

Until the ant died

Until all the clients are affected

return Assigned Map clients
Algorithm 1 describes the principle of the phase assignment. We note that each assignment_ant_agent uses the probabilistic rule to choose the depot:

$$
p_{i j}=\frac{\left(\tau 1_{i j}\right)^{\alpha}\left(\eta_{i j}\right)^{\beta}}{\sum_{d \in D}\left(\tau 1_{i d}\right)^{\alpha}\left(\eta_{i d}\right)^{\beta}} \text {, with } i \in D, j \in R
$$

$$
\eta_{\mathrm{ij}}=\frac{1}{\text { distance_depot }_{\mathrm{i}-\text { client }}} \text {, with } \mathrm{i} \in \mathrm{D}, \mathrm{j} \in \mathrm{R}
$$

where :

- $\mathrm{p}_{\mathrm{ij}}$ is the probability of the client $\mathrm{j}$ to choose the depot $i$, with $i \in D, j \in R$

- $\tau 1 \mathrm{ij}$ is the strength of pheromone trail between client $j$ and depot $i$, with $i \in D, j \in R$

- $\alpha$ is a coefficient controlling the influence of the pheromone trail $\tau 1 \mathrm{ij}$,

- $\beta$ is a coefficient controlling the influence of the visibility $\eta_{\mathrm{ij}}$

- $\mathrm{D}$ is the set of depots.

- $\mathrm{R}$ is the set of clients.

Once the colonies have finished their iterations, pheromone trails evaporate according to the formula:

$$
\tau_{\mathrm{ij}}=(1-\rho) \tau_{\mathrm{ij}},
$$

where $\rho$ is the pheromone evaporation coefficient. Moreover, the update of the pheromone trail is performed by:

$$
\begin{gathered}
\tau 1_{\mathrm{ij}}=\tau 1_{\mathrm{ij}}+\Delta \tau 1_{\mathrm{ij}}, \\
\Delta \tau 1_{\mathrm{ij}}=1 / \text { best_local_cost }_{\mathrm{d}_{\mathrm{i}}},
\end{gathered}
$$

where best_local_cost ${ }_{d_{i}}$ is the best solution found for the depot $\mathrm{i}$ in the current generation. 
The pheromone trails updated according to the best solution found in the actual generation conserve the diversity of solutions and avoid getting stuck in a local optimum [34].

\subsection{Routing phase}

In the previous phase, each client has been allocated to one of the depots, the next step is the creation of vehicle routes for each depot that can be considered as a separate vehicle routing problem VRP. We use the multi agent ant colony system for the vehicle routing problem VRP so a colony of route_ant_agents is created for each depot $d_{k}$. Each route ant agent constructs a route for vehicles which will serve the clients by beginning from depot $\mathrm{dk}$ and returning to this depot. The principle of the routing phase is described in Algorithm 2.

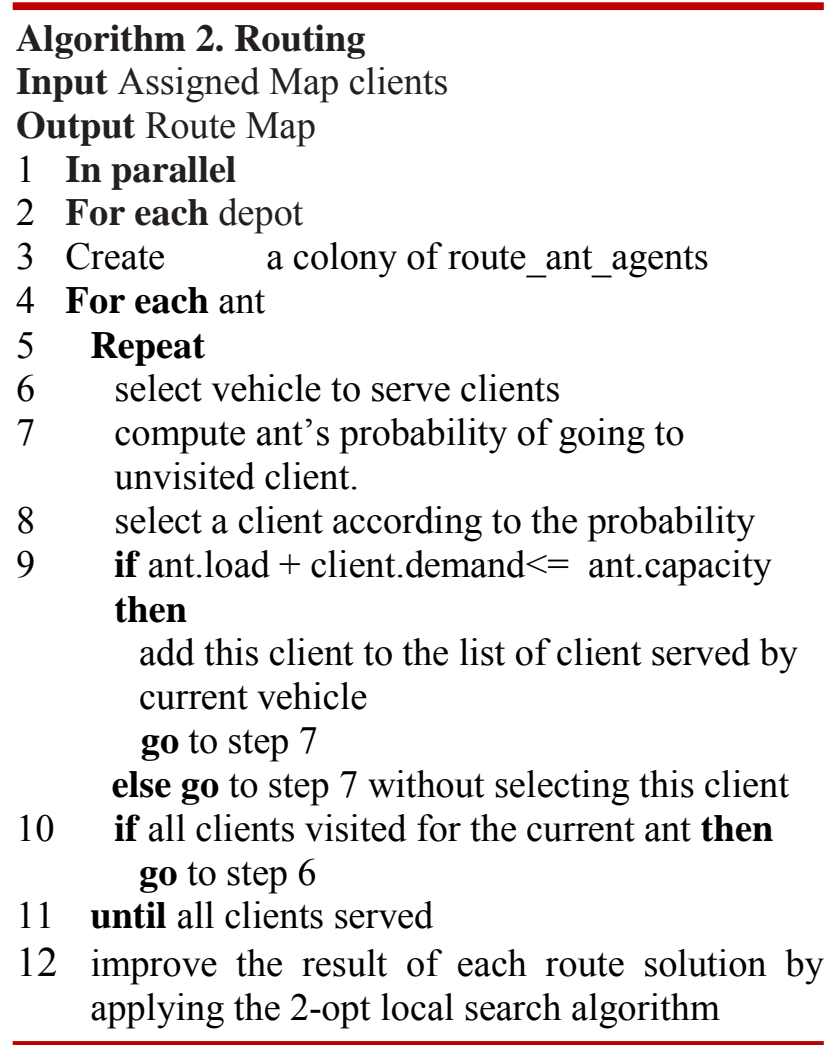

The solution shown in Algorithm 2 is improved in successive iterations. In each iteration, all route_ant_agents in the colony travel between pair of clients. The probability of an ant to visit an unvisited client is calculated based on the pheromone and the distance, and a client is chosen according to this probability.

Then the route_ant_agent checks if the ant can visit the given client (i.e., if its current load allows to add the demand of the client thereby not violating the ant's capacity). If yes, the route_ant_agent visits the specified client (adding client's demand and indicating it as selected). If not, the ant selects another unvisited node according to the calculated probability. If all clients have been visited, the ant ends the current trip with empty load (returning to its colony) and the algorithm begins a new trip. We use the following rule to choose the next client

$$
\begin{gathered}
p_{i j}=\frac{\left(\tau 2_{i j}\right)^{\alpha}\left(\eta_{i j}\right)^{\beta}}{\sum_{l \in R}\left(\tau 2_{i 1}\right)^{\alpha}\left(\eta_{i 1}\right)^{\beta}}, \\
\eta_{i j}=\frac{1}{\operatorname{cost}_{i j}} \text { with } i, j \in d_{k} \cup R,
\end{gathered}
$$

where :

- $\mathrm{p}_{\mathrm{ij}}$ is the probability of the point $\mathrm{i}$ to choose point $j$ with $i, j \in d_{k} \cup R$

- $\tau 2_{\mathrm{ij}}$ is the strength of pheromone trail between point $i$ and $j$ with $i, j \in d_{k} \cup R$

- $\alpha$ is a coefficient controlling the influence of the pheromone trail $\tau 2 \mathrm{ij}$

- $\beta$ is a coefficient controlling the influence of the visibility $\eta_{\mathrm{ij}}$

- $\mathrm{R}$ is a set of clients.

According to the following formula, the pheromone trails evaporate at the end of each iteration

$$
\tau_{i j}=(1-\rho) \tau_{i j},
$$

where $\rho$ is the pheromone evaporation coefficient

At the same time, the pheromone trail is updated according to the following rule:

$\tau 2_{\mathrm{ij}}=\tau 2_{\mathrm{ij}}+\Delta \tau 2_{\mathrm{ij}}$

where:

$\Delta \tau 2_{\mathrm{ij}}=$ best_known_solution/best_global_cost,(14) best known_solution is the best solution found of MDVRP taken from NEO web [35]

best_global_cost is the best solution found in the current generation (Equation 1).

The reactive agents have the following features:

- they are simple processing entities which can perceive their environment and react to its changes ;

- they do not use complex symbolic reasoning and do not have a symbolic representation of the world ;

- proponents-agent claim that intelligence is not a property of the active agent but it is distributed over the system and traded due to the interaction between many agents of the distributed structure and the environment.

\section{Experiments and Results}

We divide our analysis in two parts. First, we evaluate the multi-agent ant colony system on several VRP instances from [36]. Then we choose Cordeau's MDVRP instances (Inst), taken from [35], as benchmark problems for the evaluation of RMAS for MACS. 


\subsection{Vehicle Routing Problem}

This section considers the multi-agent ant colony system for the VRP, by following the steps described in Algorithm 2 (Routing), then applying two types of 1-0 exchange local search algorithm : Intra-Route and Inter-Route to generate a solution for several VRP instances from [36].

Intra-Route 1-0 exchange local search algorithm removes one client from the current route and placed it in the appropriate place in the same route. Inter-Route 1-0 exchange local search algorithm removes one client from the current route and placed it in the appropriate place of another route. Table 2 presents our results:

\begin{tabular}{cccccc}
\hline Inst. & NoV & NoC & BKS & $\begin{array}{c}\text { Before } \\
\text { L-S }\end{array}$ & $\begin{array}{c}\text { After } \\
\text { L-S }\end{array}$ \\
\hline A-n32-k5 & 5 & 32 & 784 & 808 & 784 \\
\hline A-n33-k5 & 5 & 33 & 661 & 689 & 661 \\
\hline A-n32-k6 & 6 & 32 & 742 & 753 & 742 \\
\hline A-n60-k9 & 9 & 60 & 1408 & 1619 & 1408 \\
\hline A-n80-k10 & 10 & 80 & 1764 & 2095 & 1764 \\
\hline B-n78-k10 & 10 & 78 & 1266 & 1401 & 1266 \\
\hline P-n101-k4 & 4 & 101 & 681 & 793 & 681 \\
\hline
\end{tabular}

Table 2. Results for VRP benchmark problems

\section{where}

$\mathrm{NoV}$ is the number of vehicles

$\mathrm{NoC}$ is the number of clients with the depot .

$\mathrm{L}-\mathrm{S}$ is the Local Search

We ran several tests on each instance and registered the best solution found. The proposal obtained competitive results compared with the state-of-the-art results in the majority of cases for these problem instances.

\subsection{MDVRP}

This section deals with the evaluation of the multiagent ant colony system when resolving the MDVRP, following the steps described in Figure 2. Table 3 presents our result for the MDVRP, where the last column shows the difference between our results (Our Best Solution OBS) and the best-known solutions (BKS) which were given by [35]. These best-known solutions are the objective function values defined in Equation 1, and they had been obtained by numerous algorithms throughout the history of benchmark instances.

\begin{tabular}{cccccc}
\hline Inst. & NoD & NoC & BKS & OBS & Perc-Dev \\
\hline p1 & 4 & 50 & 576.87 & 581 & 0.71 \\
\hline p2 & 4 & 50 & 473.53 & 489 & 3.26 \\
\hline p3 & 5 & 75 & 641.19 & 661 & 3.08 \\
\hline p4 & 2 & 100 & 1001.59 & 1122 & 12.02 \\
\hline p5 & 2 & 100 & 750.03 & 872 & 16.26 \\
\hline p6 & 3 & 100 & 876.5 & 930 & 6.10 \\
\hline p7 & 4 & 100 & 885.8 & 962 & 8.60 \\
\hline p8 & 2 & 249 & 4437.68 & 5151 & 16.07 \\
\hline p9 & 3 & 249 & 3900.22 & 4618 & 18.40 \\
\hline p10 & 4 & 249 & 3663.02 & 4643 & 26.75 \\
\hline p11 & 5 & 249 & 3554.18 & 4393 & 23.60 \\
\hline
\end{tabular}

Table 3. Results for MDVRP benchmark problems

where

NoD is the number of depots

NoC is the number of clients.

Perc-Dev Percentage Deviation

Table 4 compares the results obtained by our approach with other published results. Algorithms called GA1 [37], GA2 [38], and GA3 [39] are based on the principles of genetic algorithms ; and lastly, $\mathrm{ACO}$ is a different version of the algorithm based on the ACO theory [40]. The best solution values in Table 4 are computed using the objective function defined in Equation 1, and they are indicated by bold numbers.

\begin{tabular}{llllll}
\hline Inst. & Our & GA1 & GA2 & GA3 & ACO \\
\hline p1 & $\mathbf{5 8 1}$ & 591.7 & 622.2 & 598.5 & 620.5 \\
\hline p2 & 489 & 483.1 & 480.0 & 478.7 & - \\
\hline p3 & $\mathbf{6 6 1}$ & 694.5 & 706.9 & 699.2 & - \\
\hline p4 & 1122 & 1062.4 & 1024.8 & 1011.4 & 1585.9 \\
\hline p5 & 872 & 754.8 & 785.2 & - & - \\
\hline p6 & 930 & 976.0 & 908.9 & 882.5 & - \\
\hline p7 & 962 & 976.5 & 918.1 & - & 1257.9 \\
\hline p8 & 5151 & 4812.5 & 4690.2 & - & - \\
\hline p9 & 4618 & 4284.6 & 4240.1 & - & 9633.2 \\
\hline p10 & 4643 & 4291.5 & 3984.8 & - & - \\
\hline p11 & 4393 & 4092.7 & 3880.7 & - & - \\
\hline
\end{tabular}

Table 4 Best solution values obtained by various algorithms

We observe that our algorithm attempts to attain better solutions in several cases when compared to algorithms based on the genetic principle (GA1, GA2, GA3) and also in case of ACO algorithm defined in [40]. 


\begin{tabular}{llll} 
Inst. & Our & GA1 & ACO \\
\hline p1 & $\mathbf{5 8 1}$ & 591.7 & 620.5 \\
\hline p2 & 489 & 483.1 & - \\
\hline p3 & $\mathbf{6 6 1}$ & 694.5 & - \\
\hline p4 & 1122 & 1062.4 & 1585.9 \\
\hline p5 & 872 & 754.8 & - \\
\hline p6 & $\mathbf{9 3 0}$ & 976.0 & - \\
\hline p7 & $\mathbf{9 6 2}$ & 976.5 & 1257.9 \\
\hline p8 & 5151 & 4812.5 & - \\
\hline p9 & 4618 & 4284.6 & 9633.2 \\
\hline p10 & 4643 & 4291.5 & - \\
\hline p11 & 4393 & 4092.7 & - \\
\hline
\end{tabular}

Table 5 Our approach compared with GA1 and ACO algorthms

More good result when comparing with only GA1[37] and ACO [40] algorithms in table 5.

The multi-depot vehicle routing problem solved by applying the multi-ant colony system has been implemented in NetLogo [41], which is a multiagent simulation environment. In Figure 4 we can see the solution of the first instance $\mathrm{p} 1$.

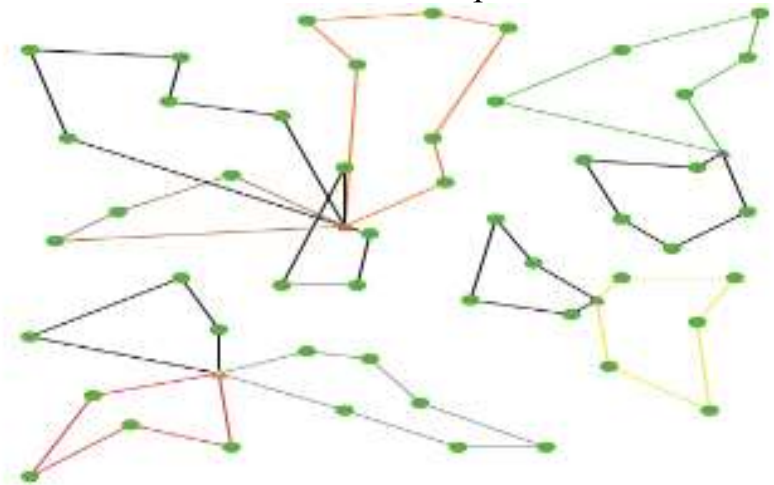

Fig. 4 The first solution instance $\mathrm{p} 1$ of the MDVRP

In order to improve the solution, we add a virtual depot to the MDVRP which convert it to a VRP, then we apply local search algorithm to the solution obtained of MDVRP converted to VRP as follows :

- Intra-Route and Inter-route 1-0 exchange local search algorithm in the same way as described in section 5.1.

- Tabu search local search algorithm.

\section{Conclusion}

In this paper, a multi-ant colony system has been proposed to solve the multi-depot vehicle routing problem MDVRP. The method has been implemented by using a reactive multi-agent system. In order to verify the algorithm, we have conducted several experiments by using VRP instances from [36] and Cordeau's MDVRP benchmark instances [35]. The obtained results have been compared with other meta-heuristic algorithms. We observe (as illustrated in Table 2 and Table 4), that the proposed algorithm provides acceptable results when compared with others.

The possibility of parallel processing is a positive feature of the proposed algorithm. In each generation, individual ants may search for solutions independently from each other. These processes are distributed using a reactive multi-agent system. Furthermore, the algorithm considers the autonomy and flexibility features of reactive agents.

The algorithm evenly offers several perspectives for future work. After the construction of the solution, the obtained results could be improved by performing a local search for the MDVRP. In the literature, there are many types of local search schemes which can be implemented to improve the solution.

\section{References :}

[1] TING, Ching-Jung et CHEN, Chia-Ho. Combination of multiple ant colony system and simulated annealing for the multidepot vehiclerouting problem with time windows. Transportation Research Record, 2008, vol. 2089, no 1, p. 85-92.

[2] DORIGO, Marco et STÜTZLE, Thomas. Ant colony optimization: overview and recent advances. In : Handbook of meta-heuristic s. Springer, Cham, 2019. p. 311-351.

[3] DORIGO, Marco et STÜTZLE, Thomas. The ant colony optimization meta-heuristic : Algorithms, applications, and advances. In : Handbook of meta-heuristic s. Springer, Boston, MA, 2003. p. 250-285.

[4] SILVA, Maria Amélia Lopes, et al. Hybrid meta-heuristic $s$ and multi-agent systems for solving optimization problems: A review of frameworks and a comparative analysis. Applied Soft Computing, 2018, 71: 433-459.

[5] MGUIS, Fethi, ZIDI, Kamel, GHEDIRA, Khaled, et al. Distributed approach for vehicle routing problem in disaster case. IFAC Proceedings Volumes, 2012, vol. 45, no 24, p. 353-359.

[6] LI, Jian; LI, Yang; PARDALOS, Panos M. Multi-depot vehicle routing problem with time windows under shared depot resources. Journal of combinatorial optimization, 2016, 31.2: 515532.

[7] STODOLA, Petr. Hybrid ant colony optimization algorithm applied to the multidepot vehicle routing problem. Natural Computing, 2020, p. 1-13.

[8] JABIR, E.; PANICKER, Vinay V.; SRIDHARAN, R. Design and development of a hybrid ant colony-variable neighbourhood 
search algorithm for a multi-depot green vehicle routing problem. Transportation Research Part D: Transport and Environment, 2017, 57: 422-457.

[9] WANG, Xinyu, et al. A novel hybrid ant colony optimization algorithm for emergency transportation problems during post-disaster scenarios. IEEE Transactions on Systems, Man, and Cybernetics: Systems, 2016, 48.4: 545-556.

[10] LIM, Andrew et WANG, Fan. Multi-depot vehicle routing problem: A one-stage approach. IEEE transactions on Automation Science and Engineering, 2005, vol. 2, no 4, p. 397-402.

[11] RAMOS, Tânia Rodrigues Pereira, GOMES, Maria Isabel, et PÓVOA, Ana Paula Barbosa. Multi-depot vehicle routing problem: a comparative study of alternative formulations. International Journal of Logistics Research and Applications, 2019, p. 1-18.

[12] YÜCENUR, G. Nilay et DEMIREL, Nihan Çetin. A new geometric shape-based genetic clustering algorithm for the multi-depot vehicle routing problem. Expert Systems with Applications, 2011, vol. 38, no 9, p. 1185911865.

[13] ESCOBAR, John Willmer, LINFATI, Rodrigo, TOTH, Paolo, et al. A hybrid granular tabu search algorithm for the multi-depot vehicle routing problem. Journal of heuristics, 2014, vol. 20, no 5, p. 483-509.

[14] LI, Jian, LI, Yang, et PARDALOS, Panos M. Multi-depot vehicle routing problem with time windows under shared depot resources. Journal of Combinatorial Optimization, 2016, vol. 31, no 2, p. 515-532.

[15] BEZERRA, Sinaide Nunes, SOUZA, Marcone Jamilson Freitas, DE SOUZA, Sérgio Ricardo, et al. A VNS-based algorithm with adaptive local search for solving the multidepot vehicle routing problem. In : International Conference on Variable Neighborhood Search. Springer, Cham, 2018. p. 167-181.

[16] JAIR, J., PATERNINA-ARBOLEDA, Carlos D., CANTILLO, Victor, et al. A twopheromone trail ant colony system-tabu search approach for the heterogeneous vehicle routing problem with time windows and multiple products. Journal of Heuristics, 2013, vol. 19, no 2, p. 233-252.

[17] TAN, Xuan, LUO, Xuyao, CHEN, W. N., et al. Ant colony system for optimizing vehicle routing problem with time windows. In : International Conference on Computational Intelligence for Modelling, Control and Automation and International Conference on
Intelligent Agents, Web Technologies and Internet Commerce (CIMCA-IAWTIC'06). IEEE, 2005. p. 209-214.

[18] GAJPAL, Yuvraj et ABAD, Prakash L. Multiant colony system (MACS) for a vehicle routing problem with backhauls. European Journal of Operational Research, 2009, vol. 196, no 1, p. 102-117.

[19] ZHANG, Shuai, ZHANG, Weiheng, GAJPAL, Yuvraj, et al. Ant colony algorithm for routing alternate fuel vehicles in multi-depot vehicle routing problem. In : Decision Science in Action. Springer, Singapore, 2019. p. 251-260.

[20] LI, Yongbo, SOLEIMANI, Hamed, et ZOHAL, Mostafa. An improved ant colony optimization algorithm for the multi-depot green vehicle routing problem with multiple objectives. Journal of cleaner production, 2019, vol. 227, p. 1161-1172.

[21] STODOLA, Petr; MAZAL, Jan. Applying the ant colony optimisation algorithm to the capacitated multi-depot vehicle routing problem. International Journal of Bio-Inspired Computation, 2016, 8.4: 228-233.

[22] PALMA-BLANCO, Andres, GONZÁLEZ, Esneyder Rafael, et PATERNINAARBOLEDA, Carlos D. A Two-Pheromone Trail Ant Colony System Approach for the Heterogeneous Vehicle Routing Problem with Time Windows, Multiple Products and Product Incompatibility. In : International Conference on Computational Logistics. Springer, Cham, 2019. p. 248-264.

[23] BOUDALI, Imen, FKI, Wajdi, et GHEDIRA, Khaled. An interactive distributed approach for the VRP with time windows. Journal of Simulation Systems, Science and Technology, 2005.

[24] DAN, Zhenggang, CAI, Linning, et ZHENG, Li. Improved multi-agent system for the vehicle routing problem with time windows. Tsinghua Science and Technology, 2009, vol. 14, no 3, p. 407-412.

[25] ZEDDINI, Besma et ZARGAYOUNA, Mahdi. Auto-organisation spatio-temporelle pour le VRPTW dynamique. Revue d'intelligence artificielle, 2011, vol. 25, no 6, p. 799-824.

[26] HASSEN, Houyem Ben, TOUNSI, Jihene, et BACHOUCH, Rym Ben. An Artificial Immune Algorithm for HHC Planning Based on MultiAgent System. 2019.

[27] YU, Bin; YANG, Z. Z.; XIE, J. X. A parallel improved ant colony optimization for multidepot vehicle routing problem. Journal of the Operational Research Society, 2011, 62.1: 183188.

[28] GAO, Junjie, et al. Automobile chain maintenance parts delivery problem using an 
improved ant colony algorithm. Advances in Mechanical Engineering, 2016, 8.9: 1687814016665297.

[29] MA, Yanfang, et al. An improved ACO for the multi-depot vehicle routing problem with time windows. In: Proceedings of the Tenth International Conference on Management Science and Engineering Management. Springer, Singapore, 2017. p. 1181-1189.

[30] LAOUID, Abdelkader, et al. A distributed multi-path routing algorithm to balance energy consumption in wireless sensor networks. Ad Hoc Networks, 2017, 64: 53-64.

[31] PILLAC, Victor, et al. A review of dynamic vehicle routing problems. European Journal of Operational Research, 2013, 225.1: 1-11.

[32] Marten SAVELSBERGH «Vehicle Routing and Scheduling》 The Logistics Institute Georgia Institute of Technology

[33] CREVIER, Benoit, CORDEAU, Jean-François, et LAPORTE, Gilbert. The multi-depot vehicle routing problem with inter-depot routes. European Journal of Operational Research, 2007, vol. 176, no 2, p. 756-773.

[34] STODOLA, Petr, et al. Using the ant colony optimization algorithm for the capacitated vehicle routing problem. In: Proceedings of the 16th International Conference on Mechatronics-Mechatronika 2014. IEEE, 2014. p. 503-510.

[35] WEB, N. E. O. Networking and Emerging Optimization. University of Malaga, Spain. Available online: http://neo. lcc. uma. es/vrp/vrp-instances/multiple-depot-vrpinstances/.

[36] http://vrp.atd-lab.inf.puc-rio.br/index.php/en/

[37] THANGIAH, Sam R. et SALHI, Said. Genetic clustering: an adaptive heuristic for the multidepot vehicle routing problem. Applied Artificial Intelligence, 2001, vol. 15, no 4, p. 361-383.

[38] OMBUKI, B. et HANSHAR, F. An effective genetic algorithm for the multi-depot vehicle routing problem. submitted, Preliminary version at Brock COSC TR CS-04-10, 2004.

[39] SUREKHA, Paneerselvam et SUMATHI, Sai. Solution to multi-depot vehicle routing problem using genetic algorithms. World Applied Programming, 2011, vol. 1, no 3, p. 118-131.

[40] CALDEIRA, Tiago Caperta Maia. Optimization of the multi-depot vehicle routing problem: an application to logistics and transport of biomass for electricity production. Technical University of Lisbon, Lisbon, 2009.

[41] WILENSKY, U. (1999).
NetLogo. http://ccl.northwestern.edu/netlogo/. Center for Connected Learning and Computer-
Based Modeling, Northwestern University, Evanston, IL

\section{Creative Commons Attribution License 4.0 (Attribution 4.0 International, CC BY 4.0)}

This article is published under the terms of the Creative Commons Attribution License 4.0 https://creativecommons.org/licenses/by/4.0/deed.en US 